\title{
MODULAR TRANSFORMATIONS OF THE ELLIPTIC HYPERGEOMETRIC FUNCTIONS, MACDONALD POLYNOMIALS, AND THE SHIFT OPERATOR
}

February, 2002

Dedicated to V. I. Arnold on his 65th birthday

\section{INTRODUCTION}

In the WZW model of conformal field theory associated with a simple complex Lie algebra $\mathfrak{g}$, one defines a holomorphic vector bundle, the bundle of conformal blocks, on the moduli space of smooth complex compact curves with marked points labeled by representations of $\mathfrak{g}$. This vector bundle comes with a projectively flat connection, see [TUY]. For curves of genus zero and one, this connection is flat and can be described in explicit classical terms. Moreover, horizontal sections admit integral representations [SV], [FV1].

For genus zero curves, the connection is the Knizhnik-Zamolodchikov (KZ) connection and the equation for horizontal sections ( $\mathrm{KZ}$ equation) is a generalization of the Gauss hypergeometric equation. The KZ equation reduces to the Gauss hypergeometric equation in a special case. The solutions to the $\mathrm{KZ}$ equations are given by integrals of certain differential forms over $m$-dimensional cycles where $m$ depends on $\mathfrak{g}$ and on the representations labeling marked points. The classical integral representation of the hypergeometric function is recovered in the case of four points on the Riemann sphere when $m=1$. The horizontal sections (conformal blocks) are holomorphic functions on

\footnotetext{
${ }^{1}$ Supported in part by NSF grant DMS-9801582.
} 
the universal covering of the configuration space of points on the complex plane and integral representation may be used to compute the action of covering transformations on conformal blocks. In this way representations of the pure braid group are obtained as monodromy representations.

We consider here the case of genus one curves with one marked point (elliptic curves) and the Lie algebra $s l_{2}$. The equation for horizontal sections is the Knizhnik-Zamolodchikov-Bernard (KZB) heat equation (11): it is essentially the heat equation associated to the Hamilton operator of a particle in a Weierstrass function potential. Again, solutions are given by generalizations of hypergeometric integrals, which are appropriately called elliptic hypergeometric integrals. Of particular interest is a finite dimensional subspace of the space of solutions, the space of conformal blocks of the WZW model. It may be characterized by symmetry and holomorphy conditions, see Sect. 2. This subspace is invariant under the (projective) action of the modular group $\mathrm{SL}(2, \mathbb{Z})$ of covering transformations of the upper half plane, viewed as the universal cover of the moduli space of elliptic curves.

We compute the projective action of the modular group on this space and relate the matrix elements of the $S$-transformation $\tau \mapsto-1 / \tau$ to values of $A_{1}$-Macdonald polynomials. This implies a special case of Kirillov's theorem, stating that the representation of the modular group on certain conformal blocks on elliptic curves for $s l_{N}$ is equivalent to a representation where the matrix elements of $S$ are given in terms of $A_{N-1}$-Macdonald polynomials.

One new feature of our computation is that it gives a formula for the matrix elements of the projective representation of the modular group (not just its conjugacy class) with respect to an explicit basis of elliptic hypergeometric integral solutions of the KZB equation (11). Moreover, the elliptic hypergeometric integrals give naturally a recursive procedure, developed in [FS] in a similar situation, to compute the matrix elements of $S$ by repeated application of the Stokes theorem. This recursive procedure shows the role of the shift operator [A] in this context: it is identical to the recursive construction of Macdonald polynomials out of Schur functions by repeated application of the shift operator. We also discuss the relation of the matrix elements of $S$ with the traces of intertwining operators of the quantum group $U_{q}\left(s l_{2}\right)$ at root of unity.

Finally, let us point out that the KZB connection is unitary with respect to a hermitian form which can also be given by integrals of elliptic hypergeometric type. They are discussed in [FG].

The authors thank P. Etingof and A. Kirillov, Jr., for useful discussions and the referee for useful suggestions. 


\section{Conformal Blocks on the Torus}

Let $\kappa$ and $p$ be non-negative integers satisfying $\kappa \geq 2 p+2$. Let $q=e^{\frac{\pi i}{\kappa}}$. Denote

$$
\begin{gathered}
{[n]_{q}=[n]=\frac{q^{n}-q^{-n}}{q-q^{-1}}, \quad[n] !=[1][2] \cdots[n], \quad\left[\begin{array}{c}
n \\
j
\end{array}\right]=\frac{[n] !}{[j] ![n-j] !},} \\
(n, q)_{j}=[n][n+1] \cdots[n+j-1] .
\end{gathered}
$$

Let $\tau \in \mathbb{C}$ be such that $\operatorname{Im} \tau>0$. The KZB-heat equation is the partial differential equation

$$
2 \pi i \kappa \frac{\partial u}{\partial \tau}(\lambda, \tau)=\frac{\partial^{2} u}{\partial \lambda^{2}}(\lambda, \tau)+p(p+1) \rho^{\prime}(\lambda, \tau) u(\lambda, \tau) .
$$

Here, the prime denotes the derivative with respect to the first argument, and $\rho$ is defined in terms of the first Jacobi theta function WW,

$$
\vartheta_{1}(t, \tau)=-\sum_{j \in \mathbb{Z}} e^{\pi i\left(j+\frac{1}{2}\right)^{2} \tau+2 \pi i\left(j+\frac{1}{2}\right)\left(t+\frac{1}{2}\right)}, \quad \rho(t, \tau)=\frac{\vartheta_{1}^{\prime}(t, \tau)}{\vartheta_{1}(t, \tau)} .
$$

Holomorphic solutions of the KZB-heat equation with the properties,

(i) $u(\lambda+2, \tau)=u(\lambda, \tau)$,

(ii) $u(\lambda+2 \tau, \tau)=e^{-2 \pi i \kappa(\lambda+\tau)} u(\lambda, \tau)$,

(iii) $u(-\lambda, \tau)=(-1)^{p+1} u(\lambda, \tau)$,

(iv) $u(\lambda, \tau)=\mathcal{O}\left((\lambda-m-n \tau)^{p+1}\right)$ as $\lambda \rightarrow m+n \tau$ for any $m, n \in \mathbb{Z}$,

are called conformal blocks associated with the family of elliptic curves $\mathbb{C} / \mathbb{Z}+\tau \mathbb{Z}$ with the marked point $z=0$ and the irreducible $s l_{2}$ representation of dimension $2 p+1$. It is known that the space of conformal blocks has dimension $\kappa-2 p-1$.

Remark. The relation of this definition of conformal blocks with the more standard one of [TUY is the following. Recall the definition of [TUY] in this case: integrable irreducible representations $V_{k, m}$ of the affine Kac-Moody Lie algebra $\widehat{s l}_{2}$ are labeled by two non-negative integers: $k$, the level and $m \in\{0, \ldots, k\}$, the highest weight. To each such pair $(k, m)$ and a curve $E_{\tau}=\mathbb{C} / \mathbb{Z}+\tau \mathbb{Z}$ one associates a space of conformal blocks: it is the space of linear functions on $V_{k, m}$ invariant under a natural action the Lie algebra $R(\tau)$ of $s l_{2}$-valued functions on $E_{\tau}$ with poles at 0 . As one varies $\tau$ in the upper half plane, the spaces of conformal blocks form a vector bundle with a flat connection. The description of this bundle and connection in terms of theta functions was explained in [FW]: the family of Lie algebras $R(\tau)$ is extended to a two parameter family $R(\lambda, \tau)$ which also acts on $V_{k, m}$. The $R(\lambda, \tau)$-invariant linear forms on $V_{k, m}$ form a vector bundle of twisted conformal blocks with a flat connection. The space of horizontal sections is then isomorphic to the space of twisted conformal blocks at any $(\lambda, \tau)$, in particular if $\lambda=0$, where (untwisted) conformal blocks are recovered. We have an injective restriction map from the space of horizontal sections of the bundle of twisted conformal blocks to the space of holomorphic functions of $\lambda, \tau$ with values in the the dual of the zero weight space $V_{m}[0] \subset V_{k, m}$ of the $2 m+1$-dimensional irreducible representation $V_{m}$ of $s l_{2}$. If $m$ 
is odd, $V_{m}[0]=0$. If $m=2 p$ is even, then $V_{m}[0]=\mathbb{C}$ and the image of the restriction map consists of functions of the form $v(\lambda, \tau)=\vartheta_{1}(\lambda, \tau) u(\lambda, \tau)$, where $u$ is a solution of the KZB-heat equation obeying (i)-(iv) with $\kappa=k+2$. Conformal blocks may also be defined in other ways which also lead to the same description in terms of theta functions: in [FG] this is done for conformal blocks defined as states of the Chern-Simons-Witten theory and in EFK] for the definition as spherical functions on the Kac-Moody group.

Introduce two transformations

$$
T u(\lambda, \tau)=u(\lambda, \tau+1), \quad S u(\lambda, \tau)=e^{-\pi i \kappa \frac{\lambda^{2}}{2 \tau}} \tau^{-\frac{1}{2}-\frac{p(p+1)}{\kappa}} u\left(\frac{\lambda}{\tau},-\frac{1}{\tau}\right),
$$

where we fix $\arg \tau \in(0, \pi)$.

Theorem 2.1. EK If $u(\lambda, \tau)$ is a solution of the KZB-heat equation, then $T u(\lambda, \tau)$ and $S u(\lambda, \tau)$ are solutions too. Moreover, the transformations $T$ and $S$ preserve the properties (i)-(iv).

The proof of Theorem 2.1 is by direct verification. Restricted to the space of conformal blocks, the transformations $T$ and $S$ satisfy the relations

$$
S^{2}=(-1)^{p} i q^{-p(p+1)} I, \quad(S T)^{3}=(-1)^{p} i q^{-p(p+1)} I,
$$

where $I$ is the identity transformation.

The modular group is the group generated by two elements $T$ and $S$ with relations

$$
S^{2}=1, \quad(S T)^{3}=1 .
$$

The modular group is naturally isomorphic to the quotient group $\operatorname{SL}(2, \mathbb{Z}) /\{ \pm I\}$. The formulas (2) define a projective representation of the modular group in the space of conformal blocks.

The isomorphism class of the projective representation of the modular group in the space of conformal blocks is described in [K] using the Kazhdan-Lusztig-Finkelberg isomorphism between the modular tensor categories arising from affine Lie algebras and the modular tensor categories arising from quantum groups at roots of unity. In this paper, we describe the action of the modular group on the space of conformal blocks using integral representations of solutions of the KZB-heat equation.

\section{Integral Representations of Solutions of the KZB-Heat Equation}

Solutions of the KZB-heat equation can be realized as elliptic hypergeometric integrals depending on parameters. Introduce special functions

$$
\sigma_{\lambda}(t, \tau)=\frac{\vartheta_{1}(\lambda-t, \tau) \vartheta_{1}^{\prime}(0, \tau)}{\vartheta_{1}(\lambda, \tau) \vartheta_{1}(t, \tau)}, \quad E(t, \tau)=\frac{\vartheta_{1}(t, \tau)}{\vartheta_{1}^{\prime}(0, \tau)} .
$$

They have the properties

$$
\begin{gathered}
E(t+1, \tau)=-E(t, \tau), \quad E(t+\tau, \tau)=-e^{-\pi i \tau-2 \pi i t} E(t, \tau), \\
\sigma_{\lambda}(t+1, \tau)=\sigma_{\lambda}(t, \tau), \quad \sigma_{\lambda}(t+\tau, \tau)=e^{2 \pi i \lambda} \sigma_{\lambda}(t, \tau) .
\end{gathered}
$$


The modular transformation properties of the functions $\sigma_{\lambda}$ and $E$ are calculated using the modular transformation properties of the function $\vartheta_{1}$,

$$
\vartheta_{1}(t, \tau+1)=e^{\frac{\pi i}{4}} \vartheta_{1}(t, \tau), \quad \vartheta_{1}\left(\frac{t}{\tau},-\frac{1}{\tau}\right)=\frac{\sqrt{-i \tau}}{i} e^{\pi i \frac{t^{2}}{\tau}} \vartheta_{1}(t, \tau)
$$

where $\sqrt{-i \tau}$ is to be interpreted by the convention $|\arg (-i \tau)|<\pi / 2$. We have

$$
\begin{aligned}
\sigma_{\lambda}(t, \tau+1) & =\sigma_{\lambda}(t, \tau), & \sigma_{\frac{\lambda}{\tau}}\left(\frac{t}{\tau},-\frac{1}{\tau}\right) & =\tau e^{-\frac{2 \pi i t \lambda}{\tau}} \sigma_{\lambda}(t, \tau), \\
E(t, \tau+1) & =E(t, \tau), & E\left(\frac{t}{\tau},-\frac{1}{\tau}\right) & =\tau^{-1} e^{\pi i \frac{t^{2}}{\tau}} E(t, \tau) .
\end{aligned}
$$

Let $\Phi_{p, \kappa}$ be the multi-valued function

$$
\Phi_{p, \kappa}=\Phi_{\kappa}\left(t_{1}, \ldots, t_{p}, \tau\right)=\prod_{j=1}^{p} E\left(t_{j}, \tau\right)^{-\frac{2 p}{\kappa}} \prod_{1 \leq i<j \leq p} E\left(t_{i}-t_{j}, \tau\right)^{\frac{2}{\kappa}} .
$$

For $\kappa \geq 2$ and $n \in \mathbb{Z}$, let $\theta_{\kappa, n}$ be the theta function of level $\kappa$

$$
\theta_{\kappa, n}(t, \tau)=\sum_{j \in \mathbb{Z}} e^{2 \pi i \kappa\left(j+\frac{n}{2 \kappa}\right)^{2} \tau+2 \pi i \kappa\left(j+\frac{n}{2 \kappa}\right) t} .
$$

We have

$$
\begin{gathered}
\theta_{\kappa, n+2 \kappa}(t, \tau)=\theta_{\kappa, n}(t, \tau), \quad \theta_{\kappa, n}(-t, \tau)=\theta_{\kappa,-n}(t, \tau) \\
\theta_{\kappa, n}\left(t+\frac{2}{\kappa}, \tau\right)=q^{2 n} \theta_{\kappa, n}(t, \tau), \quad \theta_{\kappa, n}\left(t+\frac{2 \tau}{\kappa}, \tau\right)=e^{-2 \pi i t-\frac{2 \pi i \tau}{\kappa}} \theta_{\kappa, n+2}(t, \tau) .
\end{gathered}
$$

The modular transformation properties of $\theta_{\kappa, n}$ are given by

$$
\begin{gathered}
\theta_{\kappa, n}(t, \tau+1)=q^{\frac{n^{2}}{2}} \theta_{\kappa, n}(t, \tau) \\
\theta_{\kappa, n}\left(\frac{t}{\tau},-\frac{1}{\tau}\right)=\sqrt{-\frac{i \tau}{2 \kappa}} e^{\pi i \kappa \frac{t^{2}}{2 \tau}} \sum_{m=0}^{2 \kappa-1} q^{-m n} \theta_{\kappa, m}(t, \tau) .
\end{gathered}
$$

Let $\Delta_{k} \subset \mathbb{R}^{k} \subset \mathbb{C}^{k}$ be the simplex

$$
\Delta_{k}=\left\{\left(t_{1}, t_{2}, \ldots, t_{k}\right) \in \mathbb{R}^{k} \subset \mathbb{C}^{k} \mid 0 \leq t_{k} \leq t_{k-1} \leq \cdots \leq t_{1} \leq 1\right\} .
$$

Let $\widetilde{\Delta}_{k}$ be the image of $\Delta_{k}$ under the map $\left(t_{1}, \ldots, t_{k}\right) \mapsto\left(\tau t_{1}, \ldots, \tau t_{k}\right)$. For $0 \leq k \leq p$, define

$$
J_{\kappa, n}^{[k]}(\lambda, \tau)=\int \Phi_{\kappa}\left(t_{1}, \ldots, t_{p}, \tau\right) \theta_{\kappa, n}\left(\lambda+\frac{2}{\kappa} \sum_{j=1}^{p} t_{j}, \tau\right) \prod_{j=1}^{p} \sigma_{\lambda}\left(t_{j}, \tau\right) d t_{j},
$$

where the integral is over

$$
\left\{\left(t_{1}, t_{2}, \ldots, t_{p}\right) \in \mathbb{C}^{p} \mid\left(t_{1}, \ldots, t_{k}\right) \in \Delta_{k},\left(t_{k+1}, \ldots, t_{p}\right) \in \widetilde{\Delta}_{p-k}\right\} .
$$


The integral is a meromorphic function of the exponents of $\Phi_{p, \kappa}$. It is well-defined when all of the exponents in the function $\Phi_{p, \kappa}$ have positive real parts. We are interested in the case when the exponents in the function $\Phi_{p, \kappa}$ are negative real numbers. In this case, the integral is understood as an analytic continuation from the region where the exponents have positive real parts. The branch of $\Phi_{p, \kappa}$ is determined by fixing the arguments of all factors of $\Phi_{p, \kappa}$ for the case $\tau=i$ and deforming continuously for arbitrary values of $\tau$. For $\tau=i$, we fix $\arg E\left(t_{j}, \tau\right)=0$ for $j=1, \ldots, k, \arg E\left(t_{j}, \tau\right)=\frac{\pi}{2}$ for $j=k+1, \ldots, p$, and $\arg E\left(t_{i}-t_{j}, \tau\right) \in(-\pi, \pi)$ for $1 \leq i<j \leq p$. Introduce

$$
u_{n}^{[k]}(\lambda, \tau)=u_{\kappa, n}^{[k]}(\lambda, \tau)=J_{\kappa, n}^{[k]}(\lambda, \tau)+(-1)^{p+1} J_{\kappa, n}^{[k]}(-\lambda, \tau) .
$$

Lemma 3.1. The integrals $u_{n}^{[k]}$ have the properties

$$
u_{n}^{[k]}=u_{n+2 \kappa}^{[k]}, \quad u_{n}^{[k]}=-q^{2 k(n+p-k)} u_{-n-2(p-k)}^{[k]} .
$$

Proof. The first equation is an immediate consequence of the $2 \kappa$ periodicity of the functions $\theta_{\kappa, n}$. To derive the second equation, we consider the integrals $u_{n}^{[k]}$ after the change of variables $t_{j} \mapsto-t_{j}+1$ for $1 \leq j \leq k, t_{j} \mapsto-t_{j}+\tau$ for $k+1 \leq j \leq p$. The result follows from the formulas (3), (4), (6), and (77).

Theorem 3.2. [FV1 For $0 \leq k \leq p$ and any $n$, the integrals $u_{\kappa, n}^{[k]}(\lambda, \tau)$ are solutions of the KZB-heat equation having the properties (i)-(iv).

Theorem 3.3. The set

$$
\left\{u_{n}^{[p]}(\lambda, \tau) \mid p+1 \leq n \leq \kappa-p-1\right\}
$$

is a basis for the space of conformal blocks.

Proof. We prove that for $n \in\{p+1, \ldots, \kappa-p-1\}$, the integrals $u_{n}^{[p]}$ are linearly independent over $\mathbb{C}$. In Theorem 3.7, we show that for all other values of $n$ in the interval $0 \leq n \leq \kappa$, the integrals $u_{n}^{[p]}$ are identically zero. In the limit as $\tau \rightarrow i \infty$, the leading term of $u_{n}^{[p]}$ is of the form

$$
\frac{A_{p} e^{\frac{\pi i n^{2}}{2 \kappa} \tau}}{(\sin (\pi \lambda))^{p}}\left(\left(\prod_{j=1}^{p}\left(q^{2(n+j)}-1\right)\right) B_{p}\left(\frac{n+1}{\kappa},-\frac{2 p}{\kappa}, \frac{1}{\kappa}\right) \sin (\pi \lambda(n+p))+C_{n}\left(e^{\pi i \lambda}, e^{-\pi i \lambda}\right)\right),
$$

where $C_{n}\left(e^{\pi i \lambda}, e^{-\pi i \lambda}\right)$ is a Laurent polynomial of degree $<n+p$ in $e^{\pi i \lambda}$. Here $A_{p}$ is a non-zero constant depending only on $p$, and $B_{p}(\alpha, \beta, \gamma)$ is the Selberg integral,

$$
B_{p}(\alpha, \beta, \gamma)=\frac{1}{p !} \prod_{j=0}^{p-1} \frac{\Gamma(1+\gamma+j \gamma) \Gamma(\alpha+j \gamma) \Gamma(\beta+j \gamma)}{\Gamma(1+\gamma) \Gamma(\alpha+\beta+(p+j-1) \gamma)}
$$

It follows that the $u_{n}^{[p]}$ are linearly independent provided that the coefficients $\prod_{j=1}^{p}\left(q^{2(n+j)}-\right.$ $1)$ and $B_{p}((n+1) / \kappa,-2 p / \kappa, 1 / \kappa)$ are nonzero functions for each $n$ in the range $p+1 \leq$ $n \leq \kappa-p-1$. It is straightforward to check that the product $\prod_{j=1}^{p}\left(q^{2(n+j)}-1\right)$ is 
never zero for $n$ in this interval. The Selberg integral $B_{p}((n+1) / \kappa,-2 p / \kappa, 1 / \kappa)$ is also nonzero for $n$ in this interval since $1+1 / \kappa \notin \mathbb{Z}_{\leq 0}$, and $(n-p+j) / \kappa \notin \mathbb{Z}_{\leq 0}$ for any $j$ satisfying $0 \leq j \leq p-1$.

We use the modular transformation properties of the functions $E, \sigma_{\lambda}$ and $\theta_{\kappa, n}$ to obtain the following two lemmas.

Lemma 3.4. For any $n$, the action of the operator $T$ on $u_{n}^{[p]}$ is given by the formula

$$
T u_{n}^{[p]}(\lambda, \tau)=q^{\frac{n^{2}}{2}} u_{n}^{[p]}(\lambda, \tau) .
$$

Lemma 3.5. For any $n$, we have

$$
S u_{n}^{[p]}(\lambda, \tau)=\frac{e^{-\frac{\pi i}{4}}}{\sqrt{2 \kappa}} \sum_{m=0}^{2 \kappa-1} q^{-m n} u_{m}^{[0]}(\lambda, \tau) .
$$

In Lemma 3.5, we have written $S u_{n}^{[p]}$ as a linear combination of $u_{m}^{[0]}$. Our goal is to express $S u_{n}^{[p]}$ in terms of the basis $\left\{u_{m}^{[p]}(\lambda, \tau) \mid p+1 \leq m \leq \kappa-p-1\right\}$. This is accomplished using the Stokes theorem. Repeated applications of the Stokes theorem give us a recursive procedure for expressing the integrals $u_{m}^{[0]}$ as linear combinations of the integrals $u_{m}^{[p]}$.

Lemma 3.6. For $0 \leq k \leq p-1$ and any $n$, we have

$$
[p-k]\left(q^{n+p-k}-q^{-n-p+k}\right) u_{n}^{[k]}=q^{-n-k-1}[k+1]\left(q^{-2(k+1)} u_{n+2}^{[k+1]}-u_{n}^{[k+1]}\right) .
$$

Proof. Recall that the $u_{n}^{[k]}$ are defined as analytic continuations of integrals where all of the exponents in the function $\Phi_{p, \kappa}$ have positive real parts. So the identity in the lemma relates objects which are understood as analytic continuations. To prove the identity, we begin by considering simpler objects. Namely, we consider the case when all of the exponents in $\Phi_{p, \kappa}$ have positive real parts. In this case, we can apply the Stokes theorem which gives an identity for integrals with positive exponents. Then, we analytically continue all terms of the identity to get the statement of the lemma. More precisely, let $A \subset \mathbb{C}$ be the parallelogram with vertices at $0,1, \tau, 1+\tau$. Consider the $(p+1)$-dimensional cell

$$
B_{k}=\left\{\left(t_{1}, t_{2}, \ldots, t_{p}\right) \in \mathbb{C}^{p} \mid\left(t_{1}, \ldots, t_{k}\right) \in \Delta_{k}, t_{k+1} \in A,\left(t_{k+2}, \ldots, t_{p}\right) \in \widetilde{\Delta}_{p-k-1}\right\} .
$$

Applying the Stokes theorem to $B_{k}$ gives

$$
\begin{aligned}
& \int_{\delta B_{k}} \Phi_{\kappa}\left(t_{1}, \ldots, t_{p}, \tau\right) \theta_{\kappa, n}\left(\lambda+\frac{2}{\kappa} \sum_{j=1}^{p} t_{j}, \tau\right) \prod_{j=1}^{p} \sigma_{\lambda}\left(t_{j}, \tau\right) d t_{j} \\
& \quad+(-1)^{p+1} \int_{\delta B_{k}} \Phi_{\kappa}\left(t_{1}, \ldots, t_{p}, \tau\right) \theta_{\kappa, n}\left(-\lambda+\frac{2}{\kappa} \sum_{j=1}^{p} t_{j}, \tau\right) \prod_{j=1}^{p} \sigma_{-\lambda}\left(t_{j}, \tau\right) d t_{j}=0
\end{aligned}
$$


The boundary of $B_{k}$ consists of $2 p+2$ components of dimension $p$. When all of the exponents in the function $\Phi_{p, \kappa}$ have positive real parts, the restrictions of the integrand to all but four of the boundary components are zero. Those four components are

$$
\begin{aligned}
& \gamma_{k}^{1}=\left\{\left(t_{1}, t_{2}, \ldots, t_{p}\right) \in \mathbb{C}^{p} \mid\left(t_{1}, \ldots, t_{k}\right) \in \Delta_{k}, t_{k+1} \in[0,1],\left(t_{k+2}, \ldots, t_{p}\right) \in \widetilde{\Delta}_{p-k-1}\right\}, \\
& \gamma_{k}^{2}=\left\{\left(t_{1}, t_{2}, \ldots, t_{p}\right) \in \mathbb{C}^{p} \mid\left(t_{1}, \ldots, t_{k}\right) \in \Delta_{k}, t_{k+1} \in[1,1+\tau],\left(t_{k+2}, \ldots, t_{p}\right) \in \widetilde{\Delta}_{p-k-1}\right\}, \\
& \gamma_{k}^{3}=\left\{\left(t_{1}, t_{2}, \ldots, t_{p}\right) \in \mathbb{C}^{p} \mid\left(t_{1}, \ldots, t_{k}\right) \in \Delta_{k}, t_{k+1} \in[\tau, 1+\tau],\left(t_{k+2}, \ldots, t_{p}\right) \in \widetilde{\Delta}_{p-k-1}\right\}, \\
& \gamma_{k}^{4}=\left\{\left(t_{1}, t_{2}, \ldots, t_{p}\right) \in \mathbb{C}^{p} \mid\left(t_{1}, \ldots, t_{k}\right) \in \Delta_{k}, t_{k+1} \in[0, \tau],\left(t_{k+2}, \ldots, t_{p}\right) \in \widetilde{\Delta}_{p-k-1}\right\},
\end{aligned}
$$

where $[a, b]$ denotes the straight line segment connecting $a$ and $b$. Thus, the Stokes theorem applied to $B_{k}$ gives us that the sum of the integrals over $\gamma_{1}^{k}, \gamma_{2}^{k}, \gamma_{3}^{k}$, and $\gamma_{4}^{k}$ is equal to zero.

We obtain the result of the lemma as follows. Let $B_{k}$ be defined as above. Take the analytic continuation of all terms in the identity (8). Let $\gamma_{k}^{*}$ be any component of $\delta B_{k} \backslash \cup_{i=1}^{4} \gamma_{k}^{i}$. In the case when the exponents in $\Phi_{p, \kappa}$ had positive real parts, we had that the integral over $\gamma_{k}^{*}$ was zero. Thus we must have that the analytic continuation of the integral over $\gamma_{k}^{*}$ is also zero. So we have that the sum of the integrals (understood as analytic continuations) over $\gamma_{1}^{k}, \gamma_{2}^{k}, \gamma_{3}^{k}$, and $\gamma_{4}^{k}$ is equal to zero. The integrals over the boundary components $\gamma_{1}^{k}$ and $\gamma_{4}^{k}$ are $c_{1}^{k} u_{n}^{[k+1]}$ and $c_{4}^{k} u_{n}^{[k]}$, respectively, where $c_{1}^{k}, c_{4}^{k} \in \mathbb{C}$. To recognize the integral over $\gamma_{2}^{k}$ as $c_{2}^{k} u_{n}^{[k]}$, where $c_{2}^{k} \in \mathbb{C}$, we make the change of variables $t_{k+1} \rightarrow t_{k+1}+1$. To recognize the integral over $\gamma_{3}^{k}$ as $c_{3}^{k} u_{n+2}^{[k+1]}$, where $c_{3}^{k} \in \mathbb{C}$, we make the change of variables $t_{k+1} \rightarrow t_{k+1}+\tau$. We calculate the constants $c_{j}^{k}$ using the formulas (阿), (4), and (7).

Theorem 3.7. For $0 \leq k \leq p$ and $n \in\{-p,-p+1, \ldots,-p+2 k\} \cup\{\kappa-p, \kappa-p+$ $1, \ldots, \kappa-p+2 k\}$, we have

$$
u_{n}^{[k]}=0 .
$$

Proof. We prove the statement by induction on $k$. Setting $k=0$ in Lemma 3.1 gives us the identity

$$
u_{n}^{[0]}=-u_{-n-2 p}^{[0]} .
$$

Hence, using the $2 \kappa$ periodicity of the $u_{n}^{[k]}$, for $n \equiv-p \bmod \kappa$, we have

$$
u_{n}^{[0]}=0 .
$$

Now assume the theorem is true for some $k$ in the interval from 0 to $p-1$. We must show that $u_{n}^{[k+1]}=0$ for $n \in\{-p,-p+1, \ldots,-p+2 k+2\} \cup\{\kappa-p, \kappa-p+1, \ldots, \kappa-p+2 k+2\}$. We use the reflection identity of Lemma 3.1,

$$
u_{n}^{[k+1]}=-q^{2(k+1)(n+p-k-1)} u_{-n-2(p-k-1)}^{[k+1]},
$$


and the relation of Lemma 3.6,

$$
[p-k]\left(q^{n+p-k}-q^{-n-p+k}\right) u_{n}^{[k]}=q^{-n-k-1}[k+1]\left(q^{-2(k+1)} u_{n+2}^{[k+1]}-u_{n}^{[k+1]}\right) .
$$

By the induction hypothesis, the left hand side of equation (9) is zero for $n \in\{-p,-p+$ $1, \ldots,-p+2 k\} \cup\{\kappa-p, \kappa-p+1, \ldots, \kappa-p+2 k\}$. Thus, for $n \in\{-p,-p+1, \ldots,-p+$ $2 k\} \cup\{\kappa-p, \kappa-p+1, \ldots, \kappa-p+2 k\}$, we have the recursion relations

$$
u_{n}^{[k+1]}=q^{-2(k+1)} u_{n+2}^{[k+1]} .
$$

We use the identities

$$
u_{-p+k+1}^{[k+1]}=u_{\kappa-p+k+1}^{[k+1]}=0, \quad u_{-p+k}^{[k+1]}=u_{\kappa-p+k}^{[k+1]}=0,
$$

to obtain, recursively on the subscripts, the result. The first identity is an immediate consequence of the reflection identity, and the second identity follows from comparing the recursion relations (10) for $n=-p+k$ and $n=\kappa-p+k$, respectively, with the reflection identities

$$
u_{-p+k}^{[k+1]}=-q^{-2(k+1)} u_{-p+k+2}^{[k+1]}, \quad u_{\kappa-p+k}^{[k+1]}=-q^{-2(k+1)} u_{\kappa-p+k+2}^{[p]} .
$$

Proposition 3.8. For $0 \leq k \leq p$ and any $n$,

$$
S u_{n}^{[p]}(\lambda, \tau)=\frac{e^{-\frac{\pi i}{4}}}{\sqrt{2 \kappa}}\left(\sum_{m=-p+2 k+1}^{\kappa-p-1} f_{m, n}^{(k)} u_{m}^{[k]}(\lambda, \tau)+\sum_{m=\kappa-p+2 k+1}^{2 \kappa-p-1} f_{m, n}^{(k)} u_{m}^{[k]}(\lambda, \tau)\right)
$$

where

$$
f_{m, n}^{(k)}=\frac{q^{-m(n+k)-\frac{k(k+1)}{2}}}{\left(q^{-1}-q\right)^{k}}\left[\begin{array}{l}
p \\
k
\end{array}\right]^{-1} \sum_{j=0}^{k}\left[\begin{array}{l}
k \\
j
\end{array}\right] \frac{q^{2 j n}}{(-m-p+k+1, q)_{j}(m+p-k+1, q)_{k-j}} .
$$

Proof. The proof is by induction on $k$. The result from Lemma 3.5 (where we have used the periodicity of the $u_{n}^{[k]}$ to shift the interval of summation) combined with the identity $u_{-p}^{[0]}=u_{\kappa-p}^{[0]}=0$ of Theorem 3.7 give us

$$
e^{\frac{\pi i}{4}} \sqrt{2 \kappa} S u_{n}^{[p]}(\lambda, \tau)=S^{\prime} u_{n}^{[p]}=\sum_{m=-p+1}^{\kappa-p-1} q^{-m n} u_{m}^{[0]}(\lambda, \tau)+\sum_{m=\kappa-p+1}^{2 \kappa-p-1} q^{-m n} u_{m}^{[0]}(\lambda, \tau) .
$$

But this is exactly the result for $k=0$ since

$$
f_{m, n}^{(0)}=q^{-m n} \text {. }
$$

Assuming the result for some $k$ satisfying $0 \leq k \leq p-1$, we have

$$
S^{\prime} u_{n}^{[p]}(\lambda, \tau)=\sum_{m=-p+2 k+1}^{\kappa-p-1} f_{m, n}^{(k)} u_{m}^{[k]}(\lambda, \tau)+\sum_{m=\kappa-p+2 k+1}^{2 \kappa-p-1} f_{m, n}^{(k)} u_{m}^{[k]}(\lambda, \tau) .
$$


Rewriting (11) using the identity of Lemma 3.6,

$$
u_{m}^{[k]}=\frac{q^{-m-k-1}}{\left(q^{m+p-k}-q^{-m-p+k}\right)} \frac{[k+1]}{[p-k]}\left(q^{-2(k+1)} u_{m+2}^{[k+1]}-u_{m}^{[k+1]}\right),
$$

which makes sense for all $m$ in the interval of summation since those values of $m$ never give zero denominators, we obtain

$$
\begin{aligned}
& S^{\prime} u_{n}^{[p]}(\lambda, \tau)=\frac{q^{-k-1}}{\left(q^{-1}-q\right)} \frac{[k+1]}{[p-k]} \times \\
& \left(\sum_{m=-p+2 k+1}^{\kappa-p-1} \frac{q^{-m} f_{m, n}^{(k)}}{[m+p-k]} u_{m}^{[k+1]}(\lambda, \tau)-\sum_{m=-p+2 k+3}^{\kappa-p+1} \frac{q^{-m-2 k} f_{m-2, n}^{(k)}}{[m+p-k-2]} u_{m}^{[k+1]}(\lambda, \tau)\right. \\
& \left.+\sum_{m=\kappa-p+2 k+1}^{2 \kappa-p-1} \frac{q^{-m} f_{m, n}^{(k)}}{[m+p-k]} u_{m}^{[k+1]}(\lambda, \tau)-\sum_{m=\kappa-p+2 k+3}^{2 \kappa-p+1} \frac{q^{-m-2 k} f_{m-2, n}^{(k)}}{[m+p-k-2]} u_{m}^{[k+1]}(\lambda, \tau)\right) .
\end{aligned}
$$

By Theorem 3.7, the integrals $u_{m}^{[k+1]}$ are identically zero for $m \in\{-p+2 k+1,-p+$ $2 k+2, \kappa-p, \kappa-p+1, \kappa-p+2 k+1, \kappa-p+2 k+2,2 \kappa-p, 2 \kappa-p+1\}$. Thus, we may combine the first two terms on the right hand side of (12) into one sum over $m$ in the range $-p+2 k+3 \leq m \leq \kappa-p-1$. Similarly, the last two terms give us a sum over $m$ in the range $\kappa-p+2 k+3 \leq m \leq 2 \kappa-p-1$. In each of these sums, the coefficient of $u_{m}^{[k+1]}$ is given by the expression

$$
\begin{aligned}
& \frac{q^{-m(n+k+1)-\frac{(k+1)(k+2)}{2}}}{\left(q^{-1}-q\right)^{k+1}} {\left[\begin{array}{c}
p \\
k+1
\end{array}\right]^{-1}\left(\sum_{j=0}^{k}\left[\begin{array}{l}
k \\
j
\end{array}\right] \frac{q^{2 j n}}{(-m-p+k+1, q)_{j}(m+p-k, q)_{k-j+1}}\right.} \\
&\left.+\sum_{j=1}^{k+1}\left[\begin{array}{c}
k \\
j-1
\end{array}\right] \frac{q^{2 j n}}{(-m-p+k+2, q)_{j}(m+p-k-1, q)_{k-j+1}}\right) .
\end{aligned}
$$

To complete the proof of the theorem, we must show that the expression (13) is equal to the expression

$$
\frac{q^{-m(n+k+1)-\frac{(k+1)(k+2)}{2}}}{\left(q^{-1}-q\right)^{k+1}}\left[\begin{array}{c}
p \\
k+1
\end{array}\right]^{-1} \sum_{j=0}^{k+1}\left[\begin{array}{c}
k+1 \\
j
\end{array}\right] \frac{q^{2 j n}}{(-m-p+k+2, q)_{j}(m+p-k, q)_{k-j+1}} .
$$

This is proved by direct calculation.

Corollary 3.9. For $0 \leq k \leq p-1, m \in\{-p+2 k+1, \ldots, \kappa-p-1\} \cup\{\kappa-p+2 k+$ $1, \ldots, 2 \kappa-p-1\}$, and any $n$, we have

$$
f_{m, n}^{(k+1)}=\frac{q^{-m-k-1}}{q-q^{-1}} \frac{[k+1]}{[p-k]}\left(\frac{q^{-2 k} f_{m-2, n}^{(k)}}{[m-2+p-k]}-\frac{f_{m, n}^{(k)}}{[m+p-k]}\right) .
$$


Lemma 3.10. The functions $f_{m, n}^{(k)}$ have the property

$$
f_{m, n}^{(k)}=q^{-2 k(m+p-k)+2 p n} f_{-m-2 p+2 k,-n}^{(k)} .
$$

We rewrite the sum in Proposition 3.8 using Lemmas 3.1 and 3.10 .

Corollary 3.11. For $0 \leq k \leq p$ and any $n$,

$$
S u_{n}^{[p]}(\lambda, \tau)=\frac{e^{-\frac{\pi i}{4}}}{\sqrt{2 \kappa}} \sum_{m=-p+2 k+1}^{\kappa-p-1}\left(f_{m, n}^{(k)}-q^{2 p n} f_{m,-n}^{(k)}\right) u_{m}^{[k]}(\lambda, \tau) .
$$

\section{Macdonald Polynomials and the Shift Operator}

The Macdonald polynomials of type $A_{1}$ are $x$-even polynomials in terms of $q^{m x}$, where $m \in \mathbb{Z}$. They depend on two parameters $k$ and $n$, where $k$ and $n$ are non-negative integers. They are defined by the conditions:

(1) $P_{n}^{(k)}(x)=q^{n x}+q^{-n x}+$ lower order terms, except for $P_{0}^{(k)}(x)=1$,

(2) $\left\langle P_{m}^{(k)}, P_{n}^{(k)}\right\rangle=0$ for $m \neq n$, where

$$
\langle f, g\rangle=\frac{1}{2} \text { Const Term }\left(f g \prod_{j=0}^{k-1}\left(1-q^{2(j+x)}\right)\left(1-q^{2(j-x)}\right)\right) .
$$

Example. For $n>0$, we have

$$
P_{n}^{(0)}(x)=q^{n x}+q^{-n x}
$$

The shift operator $D$ is an operator acting on functions $f(x)$ by

$$
D f(x)=\frac{f(x-1)-f(x+1)}{q^{x}-q^{-x}} .
$$

The name is "shift operator" because its action on the basic ( $q$-difference) hypergeometric functions results in a shift of the parameters [AW, Ch].

Theorem 4.1. [A] For $n \geq 1$ and $k \geq 0$, we have

$$
D P_{n}^{(k)}(x)=\left(q^{-n}-q^{n}\right) P_{n-1}^{(k+1)}(x) .
$$

Remark. It follows from Theorem 4.1 that all of the Macdonald polynomials can be calculated from the polynomials $P_{n}^{(0)}$ using the shift operator provided that $q^{-n}-q^{n} \neq 0$.

Example. For $n>0$, we have

$$
P_{n-1}^{(1)}(x)=\frac{q^{n x}-q^{-n x}}{q^{x}-q^{-x}} .
$$


5. Identification of The $f_{m, n}^{(k)}$ With VAlues of The MACDONALD POLYNOMials

Theorem 5.1. For $0 \leq k \leq p, k+1 \leq n \leq \kappa$, and $m \in\{-p+2 k+1, \ldots, \kappa-p-1\}$, we have

$$
\begin{aligned}
f_{m, n}^{(k)}-q^{2 p n} f_{m,-n}^{(k)}=q^{p n-k m-\frac{k(k+1)}{2}}\left[\begin{array}{l}
p \\
k
\end{array}\right]^{-1}\left(q^{-m-p+k}-q^{m+p-k}\right) \times \\
\left(\prod_{j=1}^{k}\left(q^{-n+j}-q^{n-j}\right)\right) P_{n-k-1}^{(k+1)}(m+p-k) .
\end{aligned}
$$

Proof. We prove the statement by induction on $k$. The case $k=0$ follows directly from the formulas

$$
f_{m, n}^{(0)}=q^{-m n}, \quad P_{n-1}^{(1)}(m+p)=\frac{q^{n(m+p)}-q^{-n(m+p)}}{q^{m+p}-q^{-m-p}} .
$$

We assume that the theorem is true for some $k$ in the range $0 \leq k \leq p-1$. To prove the theorem, we must verify the identity

$$
\begin{aligned}
& f_{m, n}^{(k+1)}-q^{2 p n} f_{m,-n}^{(k+1)}=q^{p n-(k+1) m-\frac{(k+1)(k+2)}{2}}\left[\begin{array}{c}
p \\
k+1
\end{array}\right]^{-1}\left(q^{-m-p+k+1}-q^{m+p-k-1}\right) \times \\
&\left(\prod_{j=1}^{k+1}\left(q^{-n+j}-q^{n-j}\right)\right) P_{n-k-2}^{(k+2)}(m+p-k-1)
\end{aligned}
$$

for $k+2 \leq n \leq \kappa$ and $m \in\{-p+2 k+3, \ldots, \kappa-p-1\}$. By Corollary 3.9, the left hand side of the equation (14) equals

$$
\frac{q^{-m-k-1}}{\left(q-q^{-1}\right)} \frac{[k+1]}{[p-k]}\left(q^{-2 k} \frac{f_{m-2, n}^{(k)}-q^{2 p n} f_{m-2,-n}^{(k)}}{[m+p-k-2]}-\frac{f_{m, n}^{(k)}-q^{2 p n} f_{m,-n}^{(k)}}{[m+p-k]}\right) .
$$

By Theorem 4.1, the right hand side of the equation (14) equals

$$
\begin{aligned}
q^{p n-(k+1) m-\frac{(k+1)(k+2)}{2}}\left[\begin{array}{c}
p \\
k+1
\end{array}\right]^{-1} & \left(\prod_{j=1}^{k}\left(q^{-n+j}-q^{n-j}\right)\right) \times \\
& \left(P_{n-k-1}^{(k+1)}(m+p-k)-P_{n-k-1}^{(k+1)}(m+p-k-2)\right) .
\end{aligned}
$$

Thus, the equation (14) is equivalent to the equation

$$
\begin{array}{r}
q^{-2 k} \frac{f_{m-2, n}^{(k)}-q^{2 p n} f_{m-2,-n}^{(k)}}{q^{m+p-k-2}-q^{-m-p+k+2}}+\frac{f_{m, n}^{(k)}-q^{2 p n} f_{m,-n}^{(k)}}{q^{-m-p+k}-q^{m+p-k}}=q^{p n-k m-\frac{k(k+1)}{2}}\left[\begin{array}{l}
p \\
k
\end{array}\right]^{-1} \times \\
\left(\prod_{j=1}^{k}\left(q^{-n+j}-q^{n-j}\right)\right)\left(P_{n-k-1}^{(k+1)}(m+p-k)-P_{n-k-1}^{(k+1)}(m+p-k-2)\right),
\end{array}
$$

which follows from the induction hypothesis. 
Corollary 5.2. For $0 \leq k \leq p$ and $k+1 \leq n \leq \kappa$,

$$
\begin{array}{r}
S u_{n}^{[p]}(\lambda, \tau)=\frac{e^{\frac{-\pi i}{4}}}{\sqrt{2 \kappa}} \sum_{m=-p+2 k+1}^{\kappa-p-1} q^{p n-k m-\frac{k(k+1)}{2}}\left[\begin{array}{l}
p \\
k
\end{array}\right]^{-1}\left(q^{-m-p+k}-q^{m+p-k}\right) \times \\
\left(\prod_{j=1}^{k}\left(q^{-n+j}-q^{n-j}\right)\right) P_{n-k-1}^{(k+1)}(m+p-k) u_{m}^{[k]}(\lambda, \tau) .
\end{array}
$$

The left hand side of the equation in Corollary 5.2 is equal to zero if $n=k+1, \ldots, p$ or $n=\kappa-p, \kappa-p+1, \ldots, \kappa-k-1$. This gives $2(p-k)$ relations for the $\kappa-2 k-1$ possibly nonzero functions $u_{m}^{[k]}, m=k+1, \ldots, p-k-1$.

Lemma 5.3. The $2(p-k)$ relations between the functions $u_{m}^{[k]}, m=k+1, \ldots, p-k-1$, given by Corollary 5.9 are linearly independent and thus generate all linear relations between those functions.

Proof. The coefficients of the above relations form $2(p-k)$ columns of the $S$-matrix corresponding to $\kappa^{\prime}=\kappa$ and $p^{\prime}=k$. That $S$-matrix is a non-degenerate matrix whose square is a root of unity. Thus any set of its columns is linearly independent.

\section{Representation of the Modular Group}

Let $T=\left(t_{m, n}\right)$ and $S=\left(s_{m, n}\right)$ be the matrices of the transformations $T$ and $S$, respectively, with respect to the basis

$$
\left\{u_{\kappa, n}^{[p]}(\lambda, \tau) \mid p+1 \leq n \leq \kappa-p-1\right\} .
$$

Here the matrices $T$ and $S$ are defined by

$$
\begin{aligned}
T u_{\kappa, n}^{[p]} & =\sum_{m=p+1}^{\kappa-p-1} t_{m, n} u_{\kappa, m}^{[p]}, \\
S u_{\kappa, n}^{[p]} & =\sum_{m=p+1}^{\kappa-p-1} s_{m, n} u_{\kappa, m}^{[p]} .
\end{aligned}
$$

Theorem 6.1. For $p+1 \leq m, n \leq \kappa-p-1$, we have

$$
\begin{gathered}
t_{m, n}=q^{\frac{n^{2}}{2}} \delta_{m n}, \\
s_{m, n}=\frac{e^{-\frac{\pi i}{4}}}{\sqrt{2 \kappa}} q^{p(n-m)-\frac{p(p+1)}{2}}\left(q^{-m}-q^{m}\right)\left(\prod_{j=1}^{p}\left(q^{-n+j}-q^{n-j}\right)\right) P_{n-p-1}^{(p+1)}(m),
\end{gathered}
$$

where $\delta_{m n}=1$ for $m=n$ and 0 otherwise.

The theorem follows directly from Lemma 3.4 and Theorem 5.2 . 
Example. Let $\kappa=2 p+2$. In this case, the only element of the S-matrix is

$$
s_{p+1, p+1}=\frac{(-i)^{p+1}}{\sqrt{p+1}} e^{-\pi i \frac{p+1}{4}} \prod_{j=1}^{p}\left(q^{j}+q^{-j}\right) .
$$

On the other hand, according to [FSV],

$$
u_{2 p+2, p+1}^{[p]}=a_{p} \vartheta_{1}(\lambda, \tau)^{p+1},
$$

where $a_{p}$ is a constant, and hence,

$$
S u_{2 p+2, p+1}^{[p]}=a_{p}(-i)^{p+1} e^{-\pi i \frac{p+1}{4}} \theta(\lambda, \tau)^{p+1} .
$$

This, in particular, implies

$$
\prod_{j=1}^{p}\left(q^{j}+q^{-j}\right)=\sqrt{p+1}
$$

This formula and its relations to the classical Gauss sums can be found in Ch.

The projective representation of the modular group in the space of conformal blocks described in [K] is given by the matrices $\tilde{T}=\left(\tilde{t}_{m, n}\right)$ and $\tilde{S}=\left(\tilde{s}_{m, n}\right), p+1 \leq m, n \leq$ $\kappa-p-1$, where

$$
\begin{gathered}
\tilde{t}_{m, n}=e^{-\frac{\pi i}{4}} q^{\frac{n^{2}}{2}} \delta_{m n} \\
\tilde{s}_{m, n}=\frac{i}{\sqrt{2 \kappa}} q^{-\frac{p(p+1)}{2}}\left(\prod_{j=0}^{p}\left(q^{-m+j}-q^{m-j}\right)\right) P_{n-p-1}^{(p+1)}(m) .
\end{gathered}
$$

Proposition 6.2. Let $D$ be the diagonal matrix such that

$$
d_{j}=q^{p j} \prod_{l=1}^{p}\left(q^{-j+l}-q^{j-l}\right), \quad p+1 \leq j \leq \kappa-p-1 .
$$

Then

$$
T=e^{\frac{\pi i}{4}} D^{-1} \tilde{T} D, \quad S=e^{-\frac{3 \pi i}{4}} D^{-1} \tilde{S} D
$$

Remark. For the standard definition of conformal blocks $v(\lambda, \tau)=\vartheta_{1}(\lambda, \tau)^{-1} u(\lambda, \tau)$ (see the remark in Sect. 2), the $S$ and $T$ transformations are defined as

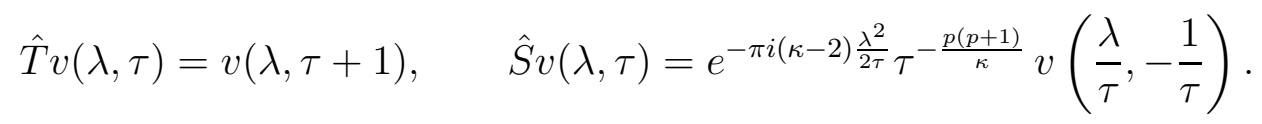

Using the transformation rules (5) of $\vartheta_{1}$ we see that the corresponding matrices $\hat{T}, \hat{S}$ are related to $T, S$ by

$$
\hat{T}=e^{-\frac{\pi i}{4}} T, \quad \hat{S}=e^{\frac{3 \pi i}{4}} S .
$$

Thus $\hat{T}, \hat{S}$ are conjugated to $\tilde{T}, \tilde{S}$. 


\section{Trace Functions for $U_{q}\left(s l_{2}\right)$}

Let $q=e^{\frac{\pi i}{\kappa}}$. The quantum group $U_{q}\left(s l_{2}\right)$ has generators $E, F, q^{c h}$, where $c \in \mathbb{C}$, with relations

$$
q^{c h} q^{c^{\prime} h}=q^{\left(c+c^{\prime}\right) h}, \quad q^{c h} E q^{-c h}=q^{2 c} E, \quad q^{c h} F q^{-c h}=q^{-2 c} F, \quad E F-F E=\frac{q^{h}-q^{-h}}{q-q^{-1}},
$$

and comultiplication defined by

$$
\Delta(E)=E \otimes q^{h}+1 \otimes E, \quad \Delta(F)=F \otimes 1+q^{-h} \otimes F, \quad \Delta\left(q^{c h}\right)=q^{c h} \otimes q^{c h} .
$$

Identify weights for $U_{q}\left(s l_{2}\right)$ with complex numbers as follows. Say that a vector $v$ in a $U_{q}\left(s l_{2}\right)$-module has weight $\nu \in \mathbb{C}$ if $q^{h} v=q^{\nu} v$. Let $M_{\mu}$ be the Verma module over $U_{q}\left(s l_{2}\right)$ with highest weight $\mu$, and let $v_{\mu}$ be its highest weight vector. Let $k$ be a non-negative integer such that $\kappa \geq 2 k+2$. Let $U$ be the irreducible finite dimensional representation of $U_{q}\left(s l_{2}\right)$ of weight $2 k$. Let $U[0]$ denote the zero weight subspace of $U$. Let $u \in U[0]$. For generic $\mu$, let $\Phi_{\mu}^{u}: M_{\mu} \rightarrow M_{\mu} \otimes U$ be the intertwining operator defined by

$$
\Phi_{\mu}^{u} v_{\mu}=v_{\mu} \otimes u+\frac{F v_{\mu}}{[-\mu]} \otimes E u+\cdots+\frac{F^{j} v_{\mu}}{[j] !(-\mu, q)_{j}} \otimes E^{j} u+\cdots .
$$

Introduce an $\operatorname{End}(U[0])$-valued function $\psi^{(k)}(q, \nu, \mu)$ defined by

$$
\psi^{(k)}(q, \nu, \mu) u=\left.\operatorname{Tr}\right|_{M_{\mu}}\left(\Phi_{\mu}^{u} q^{\nu h}\right) .
$$

Since $U[0]$ is one-dimensional, this function is a scalar function.

Theorem 7.1. EV The function $\psi^{(k)}(q, \nu, \mu)$ is given by the formula

$$
\psi^{(k)}(q, \nu, \mu)=q^{\nu \mu} \sum_{j=0}^{k}(-1)^{j} q^{\frac{j(j-3)}{2}}\left(q-q^{-1}\right)^{-j-1} \frac{[k+j] !}{[j] ![k-j] !} \frac{q^{-j \mu-(j-1) \nu}}{\prod_{l=0}^{j-1}[\mu-l] \prod_{l=0}^{j}[\nu-l]} .
$$

Introduce renormalized trace functions $\Psi^{(k)}(q, \nu, \mu)$ defined by

$$
\Psi^{(k)}(q, \nu, \mu)=\prod_{j=1}^{k}\left(\frac{q^{\mu+1-j}-q^{-\mu-1+j}}{q^{\nu+j}-q^{-\nu-j}}\right) \psi^{(k)}(q, \nu, \mu) .
$$

The function $\Psi^{(k)}$ is a holomorphic function of $\mu$.

8. IdENTIFICATION OF THE $f_{m, n}^{(k)}$ WITH VALUES OF THE TRACE FUNCTIONS $\Psi^{(k)}$

Theorem 8.1. For $0 \leq k \leq p, m \in\{-p+2 k+1, \ldots, \kappa-p-1\} \cup\{\kappa-p+2 k+$ $1, \ldots, 2 \kappa-p-1\}$, and any $n$,

$$
f_{m, n}^{(k)}=q^{p n-k m-k(k+1)}\left(q^{m+p-k}-q^{-m-p+k}\right)\left[\begin{array}{l}
p \\
k
\end{array}\right]^{-1} \Psi^{(k)}\left(q^{-1},-m-p+k,-n-1\right) .
$$


Proof. The statement of the theorem is equivalent to the identity

$$
\begin{gathered}
\left(q-q^{-1}\right)^{k} \sum_{j=0}^{k}\left[\begin{array}{l}
k \\
j
\end{array}\right](-m-p+k+j+1, q)_{k-j}(m+p-j+1, q)_{j} q^{2 j n} \\
=q^{-\frac{k(k+1)}{2}+k n} \sum_{j=0}^{k} q^{-\frac{j(j-1)}{2}-j(m+p-k+n)}\left(q-q^{-1}\right)^{j} \frac{[k+j] !}{[j] ![k-j] !} \times \\
\prod_{l=j+1}^{k}\left(q^{m+p-k+l}-q^{-m-p+k-l}\right)\left(q^{n+l}-q^{-n-l}\right) .
\end{gathered}
$$

Let $x=q^{n}$. Using the $q$-binomial theorem, we rewrite the identity (15) as

$$
\begin{aligned}
&\left(q-q^{-1}\right)^{k} \sum_{j=0}^{k}\left[\begin{array}{c}
k \\
j
\end{array}\right](-m-p+k+j+1, q)_{k-j}(m+p-j+1, q)_{j} x^{2 j-k} \\
&=q^{-k(k+1)} \sum_{j=0}^{k} q^{-j(m+p-k-1)}\left(q-q^{-1}\right)^{j} \frac{[k+j] !}{[j] ![k-j] !} \times \\
& \prod_{l=j+1}^{k}\left(q^{m+p-k+l}-q^{-m-p+k-l}\right) \sum_{i=0}^{k-j}(-1)^{k-j-i} q^{i(k+j+1)}\left[\begin{array}{c}
k-j \\
i
\end{array}\right] x^{2 i-k} .
\end{aligned}
$$

For a fixed value of $j$ in the range $0 \leq j \leq k$, the coefficient of $x^{2 j-k}$ on the left hand side of $(16)$ is

$$
\left(q-q^{-1}\right)^{k}\left[\begin{array}{l}
k \\
j
\end{array}\right](-m-p+k+j+1, q)_{k-j}(m+p-j+1, q)_{j} .
$$

The coefficient of $x^{2 j-k}$ on the right hand side of (16) is

$$
\begin{aligned}
q^{-(k+1)(k-j)}\left(q-q^{-1}\right)^{k}\left[\begin{array}{l}
k \\
j
\end{array}\right](m+p-j+1, q)_{j}(-m-p+j, q)_{k-j} \times \\
\sum_{i=0}^{k-j} q^{-i(m+p-k-j-1)} \frac{(k+1, q)_{i}(j-k, q)_{i}}{[i] !(m+p-k+1, q)_{i}} .
\end{aligned}
$$

We observe that the sum

$$
\sum_{i=0}^{k-j} q^{-i(m+p-k-j-1)} \frac{(k+1, q)_{i}(j-k, q)_{i}}{[i] !(m+p-k+1, q)_{i}}
$$

is the hypergeometric series

$$
{ }_{2} \phi_{1}\left(\begin{array}{c}
q^{-2(k+1)}, q^{-2(j-k)} \\
q^{-2(m+p-k+1)}
\end{array} ; q^{-2}, q^{-2(m+p-k-j)}\right) .
$$


The series (17) is equal to

$$
q^{(k-j)(k+1)} \frac{(m+p-2 k, q)_{k+1}}{(m+p-k-j, q)_{k+1}} .
$$

(For a proof of this formula, see [GR]). Hence the coefficients of $x^{2 j-k}$ on both sides of (16) are equal. This proves the theorem.

Under the identification in Theorem 8.1, Corollary 3.9 takes the following form.

Corollary 8.2. For $0 \leq k \leq p-1, m \in\{-p+2 k+1, \ldots, \kappa-p-1\} \cup\{\kappa-p+2 k+$ $1, \ldots, 2 \kappa-p-1\}$, and any $n$,

$$
q^{-k-1} \Psi^{(k+1)}\left(q^{-1}, m, n+k\right)=\frac{\Psi^{(k)}\left(q^{-1}, m-1, n+k\right)-\Psi^{(k)}\left(q^{-1}, m+1, n+k\right)}{q^{m}-q^{-m}} .
$$

Remark. The right hand side of the equation in Corollary 8.2 is the shift operator applied to $\Psi^{(k)}\left(q^{-1}, m, n+k\right)$. Thus, for $m$ as above, $k \geq 0$, and any $n$, we have

$$
D \Psi^{(k)}\left(q^{-1}, m, n\right)=q^{-k-1} \Psi^{(k+1)}\left(q^{-1}, m, n\right) .
$$

Together with Theorem 5.1, the identification in Theorem 8.1 gives us a formula for the Macdonald polynomials evaluated at roots of unity in terms of values of the renormalized trace functions.

Corollary 8.3. For $\kappa \geq 2 k+2, k+1 \leq n \leq \kappa$, and $m \in\{-p+2 k+1, \ldots, \kappa-p-1\}$, we have

$$
\begin{aligned}
\Psi^{(k)}\left(q^{-1},-m-p+k, n-1\right)-\Psi^{(k)}\left(q^{-1},-m\right. & -p+k,-n-1) \\
& =P_{n-k-1}^{(k+1)}(m+p-k) \prod_{j=1}^{k}\left(q^{-n+2 j}-q^{n}\right) .
\end{aligned}
$$

Remark. For a generalization of Corollary 8.3, see [ES].

Using the identification in Theorem 8.1, we have the following expression for the $S$ transformation in terms of the renormalized trace functions.

Corollary 8.4. For $0 \leq k \leq p$ and any $n$, we have

$$
\begin{aligned}
& S u_{n}^{[p]}(\lambda, \tau)=\frac{e^{-\frac{\pi i}{4}}}{\sqrt{2 \kappa}} \sum_{m=-p+2 k+1}^{\kappa-p-1} q^{p n-k m-k(k+1)}\left[\begin{array}{l}
p \\
k
\end{array}\right]^{-1}\left(q^{-m-p+k}-q^{m+p-k}\right) \times \\
&\left(\Psi^{(k)}\left(q^{-1},-m-p+k, n-1\right)-\Psi^{(k)}\left(q^{-1},-m-p+k,-n-1\right)\right) u_{m}^{[k]}(\lambda, \tau) .
\end{aligned}
$$




\section{REFERENCES}

[AI] R. Askey and M. Ismail, A generalization of ultraspherical polynomials, Studies in Pure Math. (1983), 55-78.

[AW] R. Askey and J. Wilson, Some basic hypergeometric orthogonal polynomials that generalize Jacobi polynomials, Mem. Amer. Math. Soc. 54 (1985), no. 319.

[Ch] I. Cherednik, One-dimensional double Hecke algebras and Gaussian sums, Duke Math. J. 108 (2001), no. 3, 511-538.

[EFK] P. Etingof, I. Frenkel and A. Kirillov, Jr., Spherical functions on affine Lie groups, Duke Math. J. 80 (1995), 59-90.

[EK] P. Etingof, A. Kirillov, Jr., On the affine analogue of Jack's and Macdonald's polynomials, Duke Math J. 78 (1995), no. 2, 229-256.

[ES] P. Etingof and K. Styrkas, Algebraic integrability of Macdonald operators and representations of quantum groups, Compositio Math (1998).

[EV] P. Etingof and A. Varchenko, Traces of intertwiners for quantum groups and difference equations, Duke Math J. 104 (2000), no. 3, 391-432.

[FG] F. Falceto and K. Gawędzki, Chern-Simons states at genus one, Commun. Math. Phys. 159 (1994), 549-579.

[FS] G. Felder and R. Silvotti, Modular covariance of minimal model correlation functions, Comm. Math. Phys. 123 (1989), no. 1, 1-15.

[FSV] G. Felder, L. Stevens, and A. Varchenko, Elliptic Selberg integrals, QA/0103227.

[FV1] G. Felder and A. Varchenko, Integral representations of solutions of the elliptic KnizhnikZamolodchikov-Bernard equations, Internat. Math. Res. Notices (1995), no. 5, 221-233.

[FV2] G. Felder and A. Varchenko, Three formulae for eigenfunctions of integrable Schroedinger operators, Compositio Math. 107 (1997), no. 2, 143-175.

[FW] G. Felder and C. Wieczerkowski, Conformal blocks on elliptic curves and the Knizhnik-Zamolodchikov-Bernard equations, Commun. Math. Phys. 176 (1996) 133-161

[GR] G. Gasper and M. Rahman, Basic hypergeometric series, Cambridge University Press, 1990.

$[\mathrm{K}] \quad$ A. Kirillov, Jr., On an inner product in modular tensor categories, J. Amer. Math. Soc. 9 (1996), no. 4, 1135-1169.

[SV] V. Schechtman and A. Varchenko, Arrangements of hyperplanes and Lie algebra homology, Inv. Math. 106 (1991), 139-194.

[TUY] A. Tsuchiya, K. Ueno and Y. Yamada, Conformal field theory on universal family of stable curves with gauge symmetries, Adv. Stud. Pure Math. 19 (1989), 459-566.

[WW] E. Whittaker and G. Watson, A course of modern analysis, Cambridge University Press, 1927. 